\title{
Column Parity Row Selection (CPRS) BIST Diagnosis Technique: Modeling and Analysis
}

\author{
James Chien-Mo Li, Member, IEEE, Hung-Mao Lin, and Fang-Min Wang
}

\begin{abstract}
Column Selection Row Parity (CPRS) diagnosis is an X-tolerant and low aliasing technique that is suitable for the BIST environment. A row selection LFSR randomly selects outputs of multiple scan chains so that unknowns can be tolerated. Column and row parities of selected outputs are observed to solve linear equations for the error positions. Experimental data show that CPRS achieves nearly perfect diagnosis, even in the presence of 1 percent unknowns. CPRS compresses the diagnosis data because only parities of circuit responses, instead of responses themselves, are observed. Two error distribution models (scattered and clustered) are developed and analyzed to show the effectiveness of CPRS. The analytical results are demonstrated to be accurate by more than 10,000 experiments.
\end{abstract}

Index Terms-Diagnostics, reliability, testing, fault tolerance.

\section{INTRODUCTION}

W ${ }^{\text {ITH the advance of system-on-chip technology, output }}$ response compression and built-in self test (BIST) are becoming popular test solutions for embedded cores [1], [2]. Diagnosis of BIST failures, however, is very difficult due to the constraint of compressed outputs with unknowns $(X)$ involved. The first challenging task of BIST diagnosis is to precisely identify the failing patterns and the failing locations [3]. Failing patterns are the patterns for which errors occur. An error is a mismatch between the expected good outputs and the actually observed circuit outputs. Failing locations are two-dimensional indexes of failing scan chains and failing scan cells where errors are observed. Once the failing patterns and failing locations are available, traditional scan-based diagnosis techniques (such as [4]) can then be applied to identify the faults.

Past research in BIST diagnosis can be summarized as follows: Early techniques, such as reciprocal polynomial [5] and diagonal matrix [6], can diagnose only one failing location. Observing MISR quotients or unloading MISR remainders identifies both the failing patterns and the failing locations [7], [8]. They are, however, limited in diagnosing multiple errors because of aliasing and masking problems of signature analyzers [9]. Error correcting code techniques, such as Reed-Solomon codes [10] and $\mathrm{BCH}$ codes [11], are mathematically feasible but practically too complex to be implemented. Cycling registers [12], [13], serial LFSR [14], and programmable MISR [15] enhance the diagnosis resolution by diversified polynomials. The LFSRbased random selection techniques apply repeated BIST sessions with different LFSR seeds to diagnose multiple failing locations in a chain [16], [17]. The counter-based

- The authors are with the Electrical Engineering Department and GIEE, National Taiwan University, No. 1, Sec. 4, Roosevelt Road, Taipei, Taiwan 106.E-mail:cmli@cc.ee.ntu.edu.tw.

Manuscript received 5 Jan. 2006; revised 27 July 2006; accepted 21 Aug. 2006; published online 22 Jan. 2007.

For information on obtaining reprints of this article, please send e-mail to: tc@computer.org, and reference IEEECS Log Number TC-0005-0106. selection performs binary searches for failing locations in multiple chains [3].

The abovementioned MISR-based or LFSR-based diagnosis techniques suffer from the unknowns, which is very common in BIST. Because of the MISR/LFSR feedback, one single unknown corrupts the entire signature. The first solution is to add bypass circuitry that dumps scan chain contents without compression [18], [19], [20]. Although bypass techniques are useful to diagnose failing locations, they have difficulty in searching for failing patterns. The diagnosis data volume can therefore be very large. The second solution is to continuously observe the output responses in a "streaming" way. The X-compact technique compresses outputs using an XOR network [21]. The parity technique observes the column parity and the row parity, which is cleared after unloading scan chains, so unknowns do not accumulate [22]. The convolutional compactor eliminates the feedback so unknowns disappear after a few scan cycles [23]. The above $X$-tolerant techniques are very effective in reducing the number of output pins, but their diagnosis resolutions may not be satisfactory. Specifically, if 100 scan chains are compressed into one single output, fewer than 15 percent of failing locations are correctly diagnosed in the presence of 1 percent unknowns [23].

Several improvements on the compactor-based diagnosis were recently proposed. A selective convolutional compactor masks the X-generating scan chains so that the diagnosis resolution is greatly enhanced [1]. Heuristic methods, such as [24], have been proposed to search for a particular solution, but their results can be wrong when multiple solutions exist. The compactor independent diagnosis is suitable for various kinds of compactors, such as the $\mathrm{X}$-compactor or the convolutional compactor [25]. This technique cannot be applied to the conventional MISR because of its infinite memory.

The column parity row selection (CPRS) technique has been shown to be an effective X-tolerant BIST diagnosis technique [26]. CPRS combines the advantages of the random selection technique [16] and the parity technique [22]. CPRS 


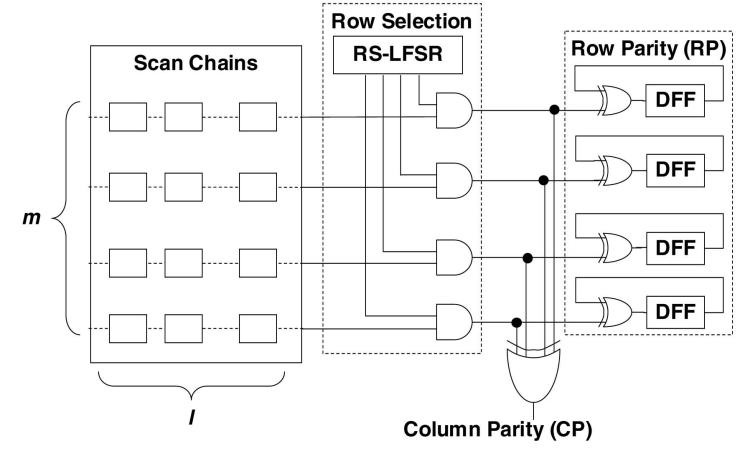

Fig. 1. CPRS hardware architecture.

provides fine diagnosis resolutions for multiple errors in multiple scan chains. What is more, CPRS solves the linear equations to find the exact solution, rather than heuristically searching for one possible solution. CPRS is X-tolerant because the row parity register is cleared after every scan unload. Experimental results show that CPRS correctly diagnoses all failing locations, even in the presence of 1 percent unknowns. On top of the abovementioned advantages, the diagnosis resolution of CPRS can be improved by additional deterministic diagnosis. Most of the past BIST diagnosis techniques show their effectiveness by experiment rather than probability analysis. This paper develops two error distribution models for analysis: the clustered model and the scattered model. The former is good for single fault, while the latter is useful for multiple faults. The probability analysis provides very good insight into the CPRS diagnosis effectiveness and helps to predict the number of sessions needed. The analytical results are demonstrated to be accurate by more than 10,000 experiments.

The organization of this paper is as follows: Section 2 introduces the CPRS hardware and its diagnosis flow. Section 3 shows the calculation methods. Sections 4 and 5 show the probabilistic analysis and experimental results, respectively. Sections 6 and 7 are discussions and summary.

\section{CPRS Hardware and Diagnosis Flow}

\subsection{Hardware Architecture}

The CPRS hardware architecture is shown in Fig. 1. During every scan cycle, the scan outputs from $m$ scan chains are randomly selected by the row selection LFSR (RS-LFSR). The row selection hardware is made up of $m$ AND gates. The selected scan outputs are XORed together to produce one bit of column parity (CP), which is observed every scan cycle. The selected scan output of every scan chain is XORed with its own row parity (RP), which is accumulated in the row parity register. The row parity is shifted out after scan chains are fully unloaded. (The row parity scan chain is not drawn in the figure for clarity.) The scan chains, the RS-LFSR, and the row parity register are all synchronized to the same clock.

\subsection{Diagnosis Flow}

The overall CPRS diagnosis flow is shown in Fig. 2. The CPRS diagnosis flow can be divided into two phases: the random diagnosis and the deterministic diagnosis. The

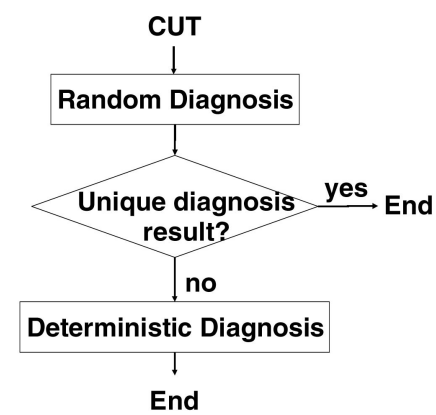

Fig. 2. Overall CPRS diagnosis flow.

circuit under test (CUT) is first diagnosed by the random diagnosis. If a unique diagnosis result is obtained, the flow is terminated; otherwise, the deterministic diagnosis is needed.

\subsubsection{Random Diagnosis}

Fig. 3 shows the steps of random diagnosis data collection. They are explained as follows:

1. Test the CUT in BIST mode with all scan chains selected. Observe $\mathrm{CP}$ and RP to identify failing patterns. The RS-LFSR outputs are ties to all ones, so all scan chains are selected.

2. Start BIST all over again and pause after a particular failing pattern is loaded.

3. An RS-LFSR seed is loaded and the row parity register is cleared.

4. Apply one system clock.

5. Unload the scan chains. The RS-LFSR and the row parity register are also clocked while unloading the scan chains. One bit of $\mathrm{CP}$ is observed every scan cycle.

6. After unloading all scan cells, the row parity is shifted out and observed.

7. If there are more sessions to go, the procedure goes back to step 2; otherwise, the random diagnosis data collection is finished.

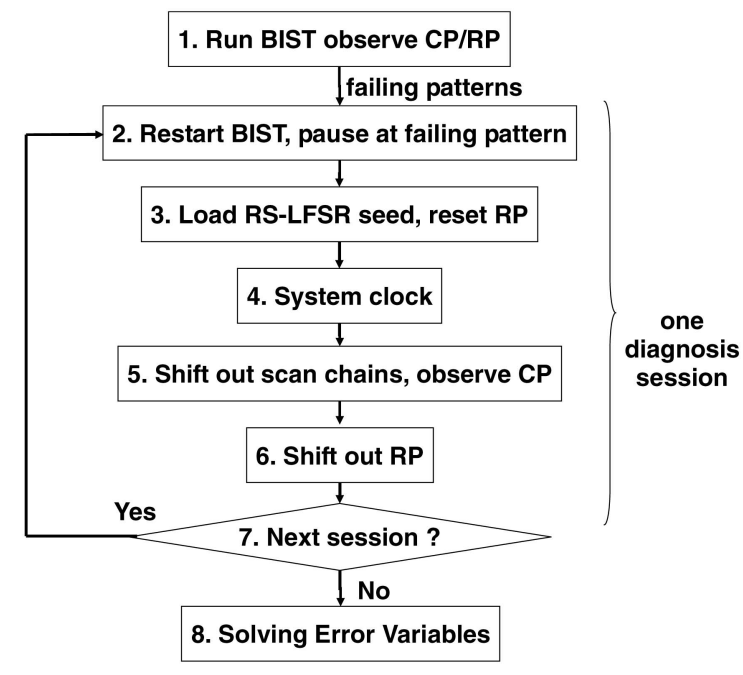

Fig. 3. Random diagnosis data collection. 
A diagnosis session consists of Steps 2 to 6 . Every diagnosis session has a distinct RS-LFSR seed. The RS-LFSR seeds are randomly generated for now because no failing locations information is available at this time. More diagnosis sessions provide finer diagnosis results at the cost of longer diagnosis time.

\subsubsection{Deterministic Diagnosis}

The deterministic diagnosis data collection is very similar to what is shown in Fig. 3. One major difference is that the RS-LFSR seed in deterministic diagnosis is calculated based on the random diagnosis results. Another difference is that deterministic diagnosis does not need to identify failing patterns, which are already known from the random diagnosis.

\subsection{Diagnosis Data Volume}

Without knowing the failing patterns, the direct dumping technique has to unload all scan chain contents after applying every pattern. Please note that binary search for failing patterns is not suitable in the BIST environment because the unknown scan outputs can corrupt the MISR signature. Therefore, the diagnosis data volume is as large as $m \times l \times P_{\text {total }}$, where $P_{\text {total }}$ is the number of patterns applied. By contrast, CPRS reduces the amount of diagnosis data because only the $\mathrm{CP}$ and $\mathrm{RP}$ are observed when identifying the failing patterns (Step 1 in Fig. 3). After that, multiple random diagnosis sessions are applied to every failing pattern. The amount of data required is therefore $(m+l) P_{\text {total }}+s(m+l) P_{\text {failing, }}$, where $s$ is the number of sessions and $P_{\text {failing }}$ is the number of failing patterns. Please note that, although random diagnosis requires multiple sessions, the total amount of data is still small because $P_{\text {failing }}$ is usually much smaller than $P_{\text {total }}$. The deterministic diagnosis is not counted here because the number of deterministic sessions is usually much smaller than that of random diagnosis.

Assume that $P_{\text {failing }}$ is much smaller than $P_{\text {total }}$ and $l$ is much larger than $m$. CPRS data volume is about $1 / m$ of the direct dumping data volume:

$$
\begin{aligned}
\frac{D A T A_{C P R S}}{D A T A_{\text {dump }}} & =\frac{(m+l) \times P_{\text {total }}+s(m+l) \times P_{\text {failing }}}{m \times l \times P_{\text {total }}} \\
& \approx \frac{m+l}{m \times l} \approx \frac{1}{m} \quad\left(P_{\text {failing }} \ll P_{\text {total }}\right) .
\end{aligned}
$$

This is a very significant compression ratio since $m$ is very large in the BIST environment. More generally,

$$
\begin{aligned}
\frac{D A T A_{C P R S}}{D A T A_{\text {dump }}} & =\frac{(m+l) \times P_{\text {total }}+s(m+l) \times P_{\text {failing }}}{m \times l \times P_{\text {total }}} \\
& \approx \frac{1+s f}{m} \quad\left(P_{\text {failing }}=f P_{\text {total }}\right),
\end{aligned}
$$

where parameter $f$ is the ratio between $P_{\text {failing }}$ and $P_{\text {total }}$ $(0<f \leq 1)$. CPRS data volume is about $(1+s f) / m$ of that of direct dumping. Still, the CPRS data volume is smaller than direct dumping as long as $s f$ is smaller than $m$. In the worst case, even if the fault under diagnosis is catastrophic and almost every pattern fails, one possible alternative solution is to unload RP after every scan clock. This is equivalent to a direct dumping of the scan chain contents.

Error Matrix
\begin{tabular}{|l|l|l|l|l|}
\hline$E_{1,1}$ & $E_{1,2}$ & $E_{1,3}$ & $E_{1,4}$ & $E_{1,5}$ \\
\hline$E_{2,1}$ & $E_{2,2}$ & $E_{2,3}$ & $E_{2,4}$ & $E_{2,5}$ \\
\hline$E_{3,1}$ & $E_{3,2}$ & $E_{3,3}$ & $E_{3,4}$ & $E_{3,5}$ \\
\hline$E_{4,1}$ & $E_{4,2}$ & $E_{4,3}$ & $E_{4,4}$ & $E_{4,5}$ \\
\hline
\end{tabular}

Fig. 4. Error matrix, selection matrix, and selected error matrix.

In this situation, the CPRS diagnosis data volume is the same as the direct dumping technique.

\section{Calculation Methods}

\subsection{Solving Error Variables}

The scan output data of a single failing pattern are represented by a two-dimensional error matrix. In the error matrix, every row corresponds to a scan chain and every element corresponds to a scan cell. If the CUT has $m$ scan chains of length $l$, then the error matrix is of size $m \times l$. Fig. 4 shows an example error matrix in which $m$ equals four and $l$ equals five. A failing scan cell in the $j$ th scan cell of the $i$ th scan chain is represented by a one in the error variable $E_{i, j}$. A passing scan cell is represented by a zero in the corresponding $E_{i, j}$.

The selection matrix $(S)$ is of the same size as the error matrix. A 1 in the selection matrix means the corresponding scan cell is selected by the row selection hardware; a 0 in the selection matrix means the corresponding scan cell is masked. The selected error matrix is obtained by ANDing the error matrix with the selection matrix. The AND operation is performed in a bit-by-bit way so the selected error matrix is also of the size $m \times l$. Fig. 4 shows an example of the selection matrix and the selected error matrix.

The error column parity $\left(C P^{E}\right)$ is a row vector of size $l$, which represents the difference between the expected good column parity and the observed column parity. A one in the $j$ th column of $C P^{E}$ means that the column parity of the $j$ th scan chain is different from its expected good value. Similarly, the error row parity $\left(R P^{E}\right)$ is a column vector of size $m$, which represents the difference of the expected good row parity and the observed row parity. Let $x$ denote the weight of $R P^{E}$ and $y$ denote the weight of $C P^{E}$. If two errors $\left(E_{1,3}\right.$ and $\left.E_{3,4}\right)$ occur in Fig. $4, C P^{E}$ and $R P^{E}$ are $\left[\begin{array}{lllll}0 & 0 & 1 & 1 & 0\end{array}\right]$ and $\left[\begin{array}{llll}1 & 0 & 1 & 0\end{array}\right]^{\mathrm{T}}$, respectively. The weights $x$ and $y$ are both 2 .

The reduced error matrix $\left(E^{R}\right)$ is obtained from the selected error matrix by deleting the rows for which the corresponding $R P^{E}$ is equal to zero and deleting the columns for which the $C P^{E}$ is equal to zero. The $E^{R}$ is therefore of size $x$ by $y$. The row equations are obtained by summing error variables of every row in the $E^{R}$. The column equations are obtained by summing all error variables of every column in the $E^{R}$.

$$
\begin{gathered}
\text { row equations }(i)=\sum_{j=1}^{c} E^{R} i, j=R P_{i}^{E}, \\
\text { column equations }(j)=\sum_{i=1}^{r} E^{R} i, j=C P_{j}^{E} .
\end{gathered}
$$




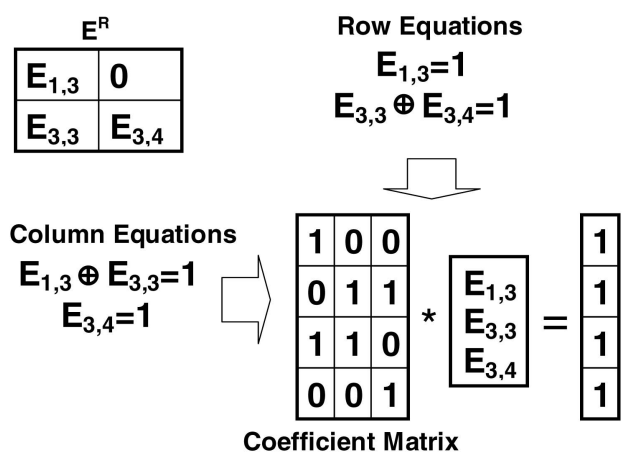

Fig. 5. Derivation of row and column equations.

A total of $x$ row equations and a total of $y$ column equations can be derived from $E^{R}$. The row equations and column equations form a system of linear equations, $A e=B$, where matrix $A$ is the coefficient matrix. The column vector $e$ represents all error variables to be solved. The column vector $B$ is the corresponding $R P^{E}$ and $C P^{E}$ for each equation. In the presence of $X \mathrm{~s}$, the row and column equations with unknown parity are deleted from the selected error matrix, so no unknowns are involved in the equations.

Fig. 5 shows an example of finding the row and column equations. The first, the second, and the fifth columns are deleted from the selected error matrix because $C P^{E}$ is $\left[\begin{array}{lllll}0 & 0 & 1 & 1 & 0\end{array}\right]$. The second and fourth rows are also deleted because $R P^{E}$ is $\left[\begin{array}{llll}1 & 0 & 1 & 0\end{array}\right]^{\mathrm{T}}$. The resulting $E^{R}$ is $2 \times 2$ in size and there are three error variables to be solved: $E_{1,3}, E_{3,3}$, and $E_{3,4}$. Two row equations and two column equations are derived from $E_{R}$. The coefficient matrix has four rows and three columns. Solving the system of linear equations produces a unique solution- $E_{1,3}=1, E_{3,3}=0$, and $E_{3,4}=1-$ which is a correct diagnosis.

If there is more than one diagnosis session, the above process is still applicable except for two changes. First, every session $s$ has its own distinct selection matrix, $C P_{s}^{E}$ and $R P_{s}^{E}$. Second, a row is deleted from the selected error matrix if no mismatch ever occurs in any one session-that is, a row is included in $E^{R}$ only if its row parity fails at least one diagnosis session. The final value of $x$ and $y$ is calculated by taking the union of $R P^{E}$ and $C P^{E}$ from all sessions, respectively.

$$
\begin{aligned}
& x=\text { weight }\left[\underset{\text { all sessions }}{\cup} R P_{s}^{E}\right], \\
& y=\text { weight }\left[\underset{\text { all sessions }}{\cup} C P_{s}^{E}\right] .
\end{aligned}
$$

After $E^{R}$ is obtained, the row and column equations of different diagnosis sessions are put together into the final linear equation system. Fig. 6 summarizes the whole flow to solve error variables for multiple sessions.

If an error variable cannot be uniquely solved, then its diagnosis result is ambiguous. Fig. 7 shows an example of the ambiguous diagnosis. The number of error variables is seven, but only six equations are available. After the GaussJordan elimination, the coefficient matrix is converted to a form of $(D \mid U)$. The upper part of $D$ is an identity matrix and

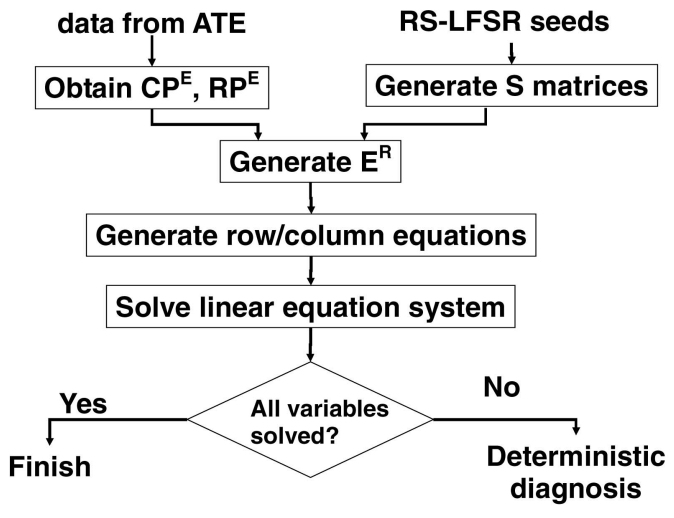

Fig. 6. Solving error variables for multiple random sessions.

the lower part of $D$ is all zeros. If the $U$ matrix is free of ones, all error variables are uniquely solved; otherwise, there are error variables that remain ambiguous. In this particular example, only one error variable, $E_{1,3}$, is uniquely solved and the other error variables are ambiguous. To diagnose the ambiguous error variables, CPRS requires more diagnosis sessions.

\subsection{Finding RS-LFSR Seed}

The RS-LFSR seeds of deterministic diagnosis are calculated based on the random diagnosis results. The purpose of deterministic diagnosis is to add new row equations and column equations which are linearly independent of the existing equations. Finding a deterministic RS-LFSR seed needs the equivalent of finding a selection matrix desired $\left(S^{M D}\right)$, which represents the specific scan cells to select. $S^{M D}$ is of the same size $(m \times l)$ as the selection matrix. A 1 in $S_{i, j}^{M D}$ represents that scan cell $i, j$ is required to be selected. A 0 in $S_{i, j}^{M D}$ represents that the corresponding scan cell should be masked. $S^{M D}$ is not unique and finding an $S^{M D}$ is similar to a covering problem. A greedy algorithm (Fig. 8) is implemented to solve this problem. Note that the greedy algorithm may not always produce the optimal solution, but it is sufficient to demonstrate the feasibility of the deterministic diagnosis.

First, $S^{M D}$ is initialized to all Xs, which stand for "not yet specified." For all unsolved error variables $E_{i, j}$, fill in zeros

\begin{tabular}{|l|l|l|}
\multicolumn{3}{|c}{$E^{R}$} \\
\hline$E_{1,3}$ & 0 & 0 \\
\hline$E_{2,3}$ & $E_{2,4}$ & $E_{2,5}$ \\
\hline$E_{3,3}$ & $E_{3,4}$ & $E_{3,5}$ \\
\hline
\end{tabular}
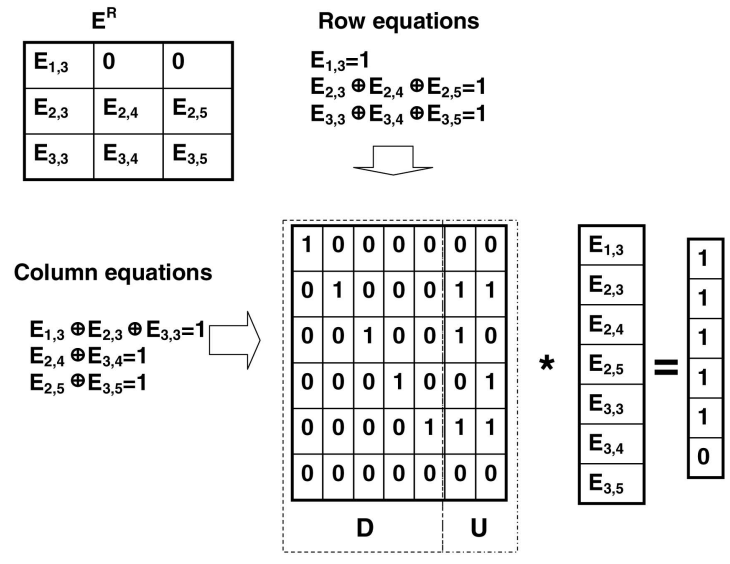

Fig. 7. Example of ambiguous diagnosis. 


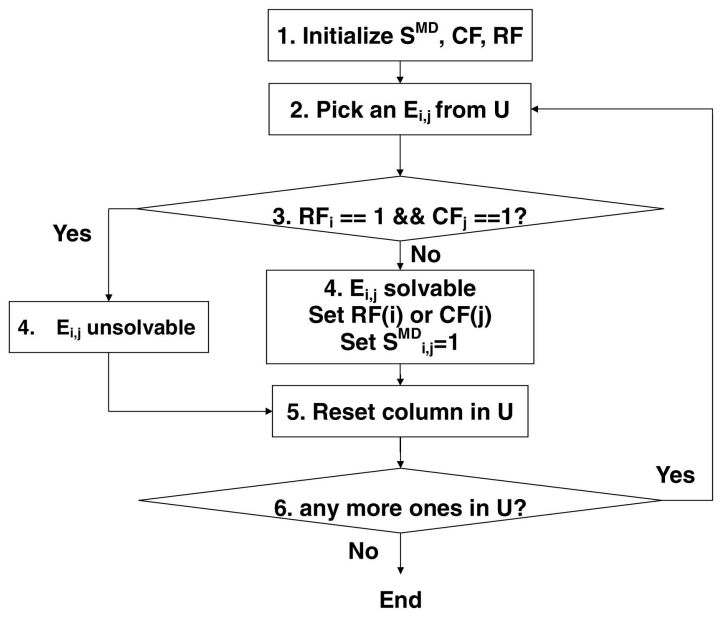

Fig. 8. Proposed method to find $S^{M D}$.

in the $i$ th row and the $j$ th column of $S^{M D}$. A column flag $(C F)$ vector and a row flag $(R F)$ vector are initialized to zeros. The $C F$ row vector is of size $l$ and the $R F$ column vector is of size $m$. A one in the $C F$ vector indicates that the corresponding column parity has been used in a column equation. A one in the $R F$ vector indicates that the corresponding row parity has been used in a row equation. Second, find a row with the smallest nonzero weight from the $U$ matrix and pick an unsolved error variable $E_{i, j}$ from that row. Third, check the $R F$ vector and the $C F$ vector of that selected error variable $E_{i, j}$. If both $\mathrm{CF}_{j}$ and $\mathrm{RF}_{i}$ are ones, which means both the $i$ th row parity and the $j$ th column parity have been used, the error variable $E_{i, j}$ is unsolvable in this deterministic diagnosis. An unsolvable error variable does not mean that it is unsolvable forever. It may become solvable in future deterministic diagnosis. If $C F_{j}$ and $R F_{i}$ are not both ones, the error variable $E_{i, j}$ is then solvable. The corresponding element $S_{i, j}^{M D}$ is set to one. Either $C F_{j}$ or $R F_{i}$ is turned from zero to one. Setting $R F_{i}$ or $C F_{j}$ to one prevents the corresponding row or column parity from being reused by the subsequent unsolved error variables. In the fifth step, label $E_{i, j}$ is solved by setting all bits in the $E_{i, j}$ column of the $\mathrm{U}$ matrix to 0 s. If there is any 1 in the U matrix, go back to Step 2; otherwise, the greedy algorithm finishes with an $S^{M D}$.

Fig. 9 demonstrates an example to find $S^{M D}$. Initially, all five unsolved error variables are initialized to $0 \mathrm{~s}$ in $S^{M D}$. The error variable $E_{2,5}$ is first picked because the second

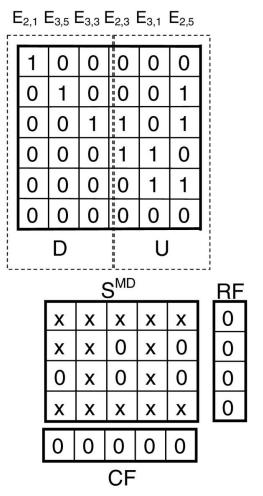

(a)
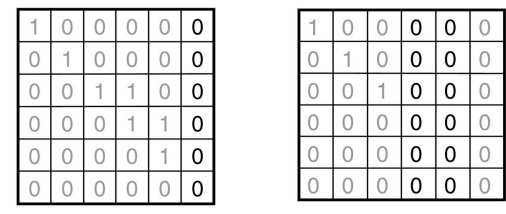

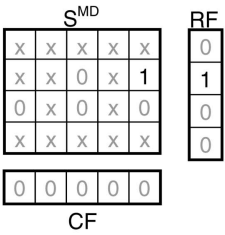

(b)

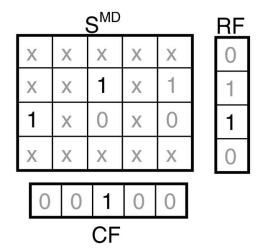

(c)
Fig. 9. Example to find $\mathrm{S}^{\mathrm{MD}}$. (a) Initial. (b) After first iteration. (c) After third iteration.

row of $\mathrm{U}$ has the smallest nonzero row weight. Since neither $R F_{2}$ nor $C F_{5}$ is 1 , this error variable is solvable. The corresponding $S_{2,5}^{M D}$ is set to one and the $R F_{2}$ is also set to one. The whole column six in the $U$ matrix is cleared to 0 s. The second error variable picked is $E_{2,3}$. Since $R F_{2}$ has already been one, $C F_{3}$ is now set to one. The fourth column in the $U$ matrix is cleared. In the last iteration, $E_{3,1}$ is set to 1 and $R F_{3}$ is set to 1 . After three iterations, there are no more 1 s in the $U$ matrix and the final $S^{M D}$ is obtained.

Once the $S^{M D}$ matrix is obtained, the corresponding RS-LFSR seed can be solved. Solving LFSR seeds has been published in previous papers, such as [27]. An example to solve the RS-LFSR is provided in Fig. 10. The black cells are to be masked (corresponding to $E_{3,3}$ and $E_{3,5}$ in $S^{M D}$ ) and the white scan cells are to be selected. The other gray cells are don't cares. Suppose that the initial contents in the fourstage RS-LFSR are $S_{0}, S_{1}, S_{2}$, and $S_{3}$. Based on the feedback of this LFSR, a system of linear equations can be derived

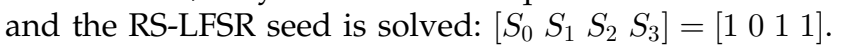

It has been shown that the chance of success is very high as long as the number of care bits is smaller than the number of LFSR stages by 20 [28]. In other words, the chance of finding an RS-LFSR seed is high as long as enough random sessions are applied with very few unsolved error variables left. In case no RS-LFSR seed is found to solve all error variables, two solutions are available: adding more deterministic sessions or adding more random sessions. For the former solution, unsolved error variables are divided into groups, which are solved one group by one group in multiple deterministic diagnosis

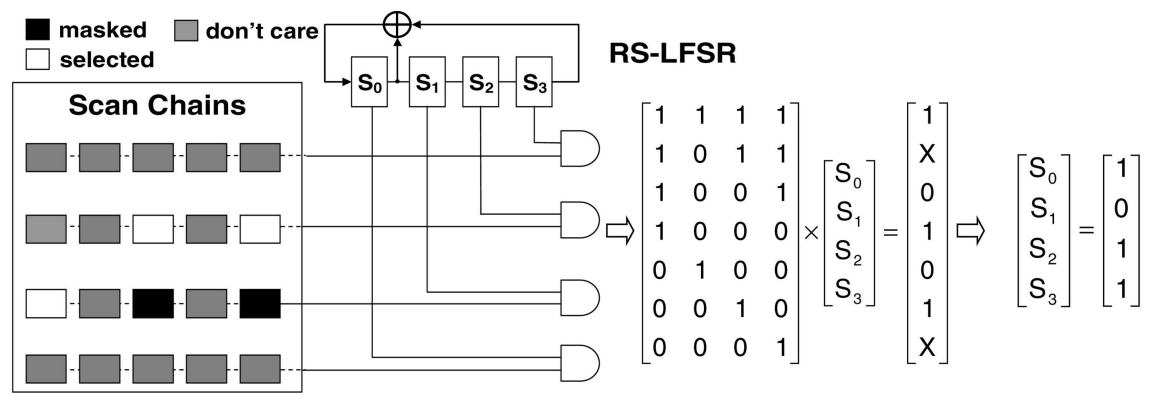

Fig. 10. Example of finding RS-LFSR seed. 


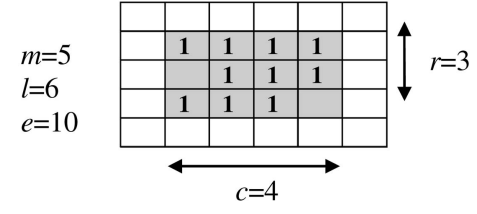

Fig. 11. Clustered errors and ROI.

sessions. The drawback of multiple deterministic sessions is that it requires iterative data collection. To avoid the iterative data collection, it is desirable to have a guideline to determine the number of random sessions, which is presented in the next session.

\section{Probability Analysis}

Two models are proposed to analyze the effectiveness of the CPRS diagnosis: the clustered model and the scattered model. The former assumes that errors are spatially confined within a small number of rows and columns; the latter assumes that errors are uniformly distributed over all rows and columns.

\subsection{Clustered Model}

\subsubsection{Assumptions}

Real silicon data have shown that defects can cluster together on the die [29]. These clustered defects can manifest themselves as clustered errors which are physically very close to each other. The clustered model is proposed here to diagnose circuits with clustered defects. Fig. 11 shows an example error matrix of clustered defects. Errors are clustered in a region of interest (ROI) sized $r \times c$, where $r$ is the number of erroneous rows and $c$ is the number of erroneous columns. The error multiplicity, $e$, represents the total number of errors in the ROI. For clustered defects, it is reasonable to assume that the number $r$ is much smaller than the number $c$, which means that errors are located very close to each other. A detailed discussion of the defect distribution is out of the scope of this paper. Interested readers can refer to [29] for more information.

To simplify the probability analysis, every error variable is assumed to be selected by an independent probability of $1 / 2$. It follows that the probability of identifying an erroneous row and the probability of identifying an erroneous column are both $1 / 2$. The number of rows and columns included in $E^{R}$ up to the sth session are represented by random variables $x$ and $y$, respectively. Because an erroneous row is included in $E^{R}$ only if its row parity fails at least once during the $s$ diagnosis sessions, the random variable $x$ obeys the binomial distribution with parameters $n_{x}=r$ and $p=\left(1-2^{-s}\right)$. Similarly, random variable $y$ obeys the binomial distribution with parameters $n_{y}=c$ and $p=\left(1-2^{-\mathrm{s}}\right)$. The random variables $x$ and $y$ are assumed to be independent.

\subsubsection{Expected Number of Correctly Diagnosed Bits}

For the clustered model, the expected number of correctly diagnosed bits up to the sth session is given by

$$
E C_{\text {cluster }}(s)=m \times l-v(s)+u(s)-\varepsilon(s),
$$

where $m \times l$ is the total number of bits in the whole error matrix. $v(s)$ represents the expected number of error variables included in $E^{R}$, out of which $u(s)$ are uniquely solved. $\varepsilon(s)$ is the expected number of errors that escape all $s$ diagnosis sessions.

The expected value of $x$ is $\mathrm{E}[x]=n_{x} p=r\left(1-2^{-\mathrm{s}}\right)$, which represents the average number of rows in $E^{R}$ up to the $s$ th session. (Please distinguish the symbol of expected value from the symbol of errors.) Similarly, the expected value of $y$ is $\mathrm{E}[\mathrm{y}]=n_{y} p=c\left(1-2^{-\mathrm{s}}\right)$, which represents the average number of columns in $E^{R}$. The expected number of error variables included in $E^{R}$ up to the $s$ th session is given by

$$
v(s)=\mathrm{E}[x y]=\mathrm{E}[x] \mathrm{E}[y]=c r\left(1-2^{-s}\right)^{2} .
$$

The expected number of row equations and column equations up to the sth session is $q(s)$, which is $s \mathrm{E}[x+y]=s(c+r)\left[1-2^{-\mathrm{s}}\right]$. Out of these $q(s)$ equations, $q_{i}(s)$ of them are linearly independent. The exact value of $q_{i}(s)$ is difficult to calculate in an analytical form, so the following linear approximation is made:

$$
q_{i}(s) \approx \frac{q(s)}{\alpha},
$$

where $\alpha$ is a fitting parameter between 2 and 3 in a typical CPRS diagnosis environment. The linear approximate will be validated by experiments in the coming subsection. Finally, the number of uniquely solved error variables, $u(s)$, is approximately equal to the number of linear independent equations $q_{i}(s)$ or the number of error variables $v(s)$, whichever is the minimum.

$$
u(s) \approx \min \left[q_{i}(s), v(s)\right] .
$$

An error is not included in $E^{R}$ if its row parity or its column parity never fails in any of the $s$ sessions. Assuming two events are independent, the probability of each event is $2^{-\mathrm{s}}$ and the probability of both events is $4^{-\mathrm{s}}$. The expected number of errors escaping the diagnosis is, therefore, as follows:

$$
\varepsilon(s)=e\left[2 \times 2^{-s}-4^{-s}\right] .
$$

To have a good diagnosis, the last three terms in (7) are close to zero. Assuming that $s$ is large and the $2^{-\mathrm{s}}$ term is ignored, the following condition is required for a good CPRS diagnosis:

$$
s_{\text {cluster }} \geq \alpha \times\left(\frac{c r}{c+r}\right) .
$$

Equation (12) provides a criterion to estimate the number of random diagnosis sessions needed. It indicates that the effectiveness of CPRS depends only on the error distribution, not on the CUT size. This is a very promising result because the number of CPRS diagnosis sessions needed does not increase with CUT size. It is also observed that the effectiveness of CPRS does not change with the RS-LFSR weight. This is because, as the weight factor increases, the decrease in $\varepsilon(s)$ is cancelled by the increase in $v(s)$. Tuning the RS-LFSR weight higher than $1 / 2$, on the one hand, gives the benefit of obtaining more equations, but, on the other 


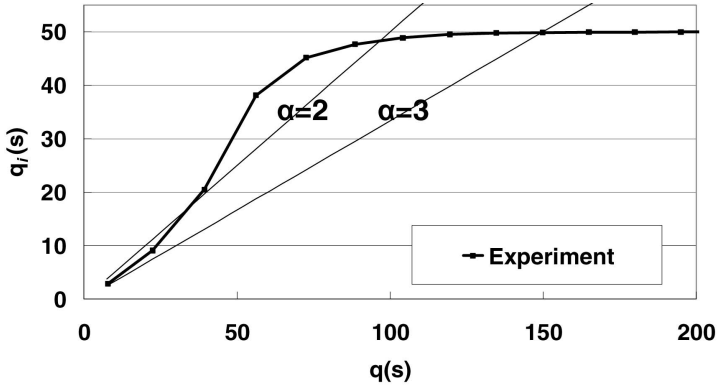

Fig. 12. Validation of linear assumption.

hand, has the drawback of involving more error variables. Eventually, the number of sessions needed does not change with the biased RS-LFSR.

\subsubsection{Validation of Linear Approximation}

Experiments are performed to validate the linear approximation in (9). In each experiment, a selection matrix is generated by an RS-LFSR with random seeds. The number of independent equations, $q_{i}(s)$, is accumulated as a function of $s$. Fig. 12 shows the average number of $q_{i}(s)$ over 10,000 experiments. The size of the ROI is $5 \times 10$, so the maximum value of $q_{i}(s)$ is 50 . The expected number of error multiplicity is 40 (i.e., 80 percent of the ROI is randomly filled with errors). Although $q_{i}(s)$ is not actually linear with respect to $q(s)$, the linear assumption of parameters $\alpha=2$ or 3 gives a reasonably close estimation. $\alpha=2$ is better for a small number of sessions and $\alpha=3$ is better for a large number of sessions.

\subsection{Scattered Model}

\subsubsection{Assumptions}

Real diagnosis data have shown that multiple faults (possibly caused by multiple defects) do occur on defective chips [30]. The scattered model is proposed to take into account the effect of multiple defects that are not clustered together. Analysis of the scattered model is more complicated than that of the clustered model because little error location information is available. To simplify the analysis, it

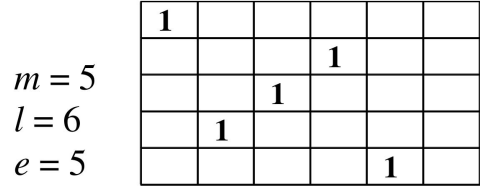

Fig. 13. Nonsharing spatial distribution.

is assumed that no error shares a column. This assumption is legitimate because $l$ is much greater than $m$ and the probability of multiple errors sharing a column is much smaller than the probability of multiple errors sharing a row. It is also assumed that the error multiplicity is small enough (say, $e<2 m$ ) that the possibility of more than four errors sharing a row is very low.

\subsubsection{Expected Number of Correctly Diagnosed Bits}

For the scattered model, the expected number of correctly diagnosed bits up to the sth session is given by

$$
E C_{\text {scatter }}(s)=m \times l-\sum_{\text {all } d} \mathrm{SP}(d) \times[v(d, s)-u(d, s)+\varepsilon(d, s)],
$$

where $d$ represents the spatial distribution of errors and $S P(d)$ is the spatial probability of $d . S P(d)$ stands for the probability that a specific spatial distribution $d$ appears. Equation (13) is similar to (7) except that the $v(d, s), u(d, s)$, and $\varepsilon(d, s)$ are now dependent on the spatial distribution $d$ as well as $s$.

Fig. 13 shows an example nonsharing spatial distribution. In the nonsharing spatial distribution, every error occupies a column and every error occupies a row-that is, no error shares a row or a column. The spatial probability of the nonoverlapping distribution is shown in the first row in Table 1. In this specific spatial distribution, random variables $x$ and $y$ are identical binomial random variables with parameters $n=e$ and $p=\left[1-2^{-s}\right] . x$ and $y$ are identical because, when an error is selected, both its CP and RP are erroneous. The expected numbers of error

TABLE 1

Seven Most Frequent Spatial Distributions

\begin{tabular}{|l|l|l|l|l|l|}
\hline$d$ & description & $\mathrm{SP}(d)$ & $v(d, s)$ & $q(d, s)$ & $\varepsilon(d, s)$ \\
\hline 1 & $\begin{array}{l}\text { Non-sharing } \\
\text { (No error shares a row.) }\end{array}$ & $C_{e}^{m} e ! / m^{e}$ & $e\left[1-2^{-S}\right] 2^{-S}+e^{2}\left[1-2^{-S}\right]^{2}$ & $2 s e\left[1-2^{-S}\right]$ & $e 2^{-S}$ \\
\hline 2 & $\begin{array}{l}\text { Only one pair of errors } \\
\text { shares one row. }\end{array}$ & $C_{1}^{m} C_{2}^{e} C_{e-2}^{m-1}(e-2) ! / m^{e}$ & $<e(e-1)\left[1-2^{-S}\right]$ & $>2 s(e-1)\left[1-2^{-S}\right]$ & $\approx e 2^{-S}$ \\
\hline 3 & $\begin{array}{l}\text { Only two pairs of errors } \\
\text { share two rows. }\end{array}$ & $C_{2}^{m} C_{4}^{e} C_{2}^{4} C_{e-4}^{m-2}(e-4) ! / m^{e}$ & $<e(e-2)\left[1-2^{-S}\right]$ & $>2 s(e-2)\left[1-2^{-S}\right]$ & $\approx e 2^{-\mathrm{S}}$ \\
\hline 4 & $\begin{array}{l}\text { Only three errors share one } \\
\text { row. }\end{array}$ & $C_{1}^{m} C_{3}^{e} C_{e-3}^{m-1}(e-3) ! / m^{e}$ & $<e(e-2)\left[1-2^{-S}\right]$ & $>2 s(e-2)\left[1-2^{-S}\right]$ & $\approx e 2^{-\mathrm{S}}$ \\
\hline 5 & $\begin{array}{l}\text { Only four errors share one } \\
\text { row. }\end{array}$ & $C_{1}^{m} C_{4}^{e} C_{e-4}^{m-1}(e-4) ! / m^{e}$ & $<e(e-3)\left[1-2^{-S}\right]$ & $>2 s(e-3)\left[1-2^{-S}\right]$ & $\approx e 2^{-\mathrm{S}}$ \\
\hline 6 & $\begin{array}{l}\text { Two errors share a row and } \\
\text { three errors share another } \\
\text { row. }\end{array}$ & $C_{1}^{m} C_{3}^{e} C_{1}^{m-1} C_{2}^{e-3} C_{e-5}^{m-2}(e-5) ! / m^{e}$ & $<e(e-3)\left[1-2^{-S}\right]$ & $>2 s(e-3)\left[1-2^{-S}\right]$ & $\approx e 2^{-S}$ \\
\hline 7 & $\begin{array}{l}\text { Only three pairs of errors } \\
\text { share a row. }\end{array}$ & $C_{3}^{m} C_{6}^{e} C_{2}^{6} C_{2}^{4} C_{e-6}^{m-3}(e-6) ! / m^{e}$ & $<e(e-3)\left[1-2^{-S}\right]$ & $>2 s(e-3)\left[1-2^{-S}\right]$ & $\approx e 2^{-\mathrm{S}}$ \\
\hline
\end{tabular}


variables included in the reduced error matrix $-v(d, s)$-is $\mathrm{E}\left[x^{2}\right]$. The value of $v(d, s)$ is calculated by the fact that the second moment of a binomial random variable is $n p(1-p)+n^{2} p^{2}$. The expected number of row and column equations- $q(d, s)$-is $2 s$ times $\mathrm{E}[x]$. The expected number of escaped errors is $\varepsilon(d, s)=n(1-p)=e 2^{-s}$.

Following the same procedure as the clustered model, the minimum number of sessions needed for a good diagnosis is as follows:

$$
s_{\text {nonsharing }} \geq \alpha \times\left(\frac{e^{2}}{2 e}\right)=\alpha \times \frac{e}{2} .
$$

The linear assumption states that $q_{i}(d, s)=q(d, s) / \alpha$. The fitting parameter $\alpha$ of the scattered model is also between 2 and 3 according to our experiments. Equation (14) indicates that, in the scatter model, the number of sessions needed is about 1 to 1.5 times as many as the error multiplicity.

In addition to the nonsharing spatial distribution, there are many other sharing spatial distributions. The sharing spatial distributions have at least two errors that share one row. Table 1 lists the six most frequent sharing spatial distributions $(d=2$ to 7$)$. These spatial distributions are mutually exclusive and their spatial probabilities sum up to nearly one as long as $e$ is smaller than $2 m$. For the sharing spatial distribution, $x$ and $y$ are not identical, so the exact values of $v(d, s)=\mathrm{E}[x y]$ are hard to calculate. Nevertheless, the upper bound of $\mathrm{E}[x y]$ is $e$ times $\mathrm{E}[x]$ because $y$ is always smaller than $e$. The $q(d, s)=s \mathrm{E}[x+y]$ is lower bounded by $2 s$ times $\mathrm{E}[x]$ because $x$ is always smaller than $y . \varepsilon(d, s)$ of the sharing spatial distributions is approximated by that of the nonsharing spatial distribution.

\subsection{Considering Unknowns}

An unknown that shares the same row with at least one error is called a row sharing unknown. An unknown that shares the same column with at least one error is called a column sharing unknown. An unknown that shares neither row nor column with any error does not cause diagnosis problems because its associated row/column equations can be discarded without penalty. Only the row sharing unknowns or column sharing unknowns cause diagnosis degradation. The distribution of unknowns and errors can be classified into four cases: scattered errors scattered unknowns, clustered errors scattered unknowns, scattered errors clustered unknowns, and clustered errors clustered unknowns.

In the first and the second cases, it is assumed that the frequency of row sharing unknowns is much higher than the frequency of column sharing unknowns. Similarly to the scattered errors, scattered unknowns also have various spatial distributions. Two modifications in $E C(s)$ are therefore proposed to consider the effect of $X$. The number of variables involved in the presence of unknowns, $v_{X}(s)$, can be approximated by

$$
v_{X}(s) \approx v(s)-\frac{r_{X} e 2^{-s}}{2},
$$

where $r_{X}$ is the average number of row sharing unknowns. The last term in (15) represents the number of error variables that are not included due to the unknowns. The

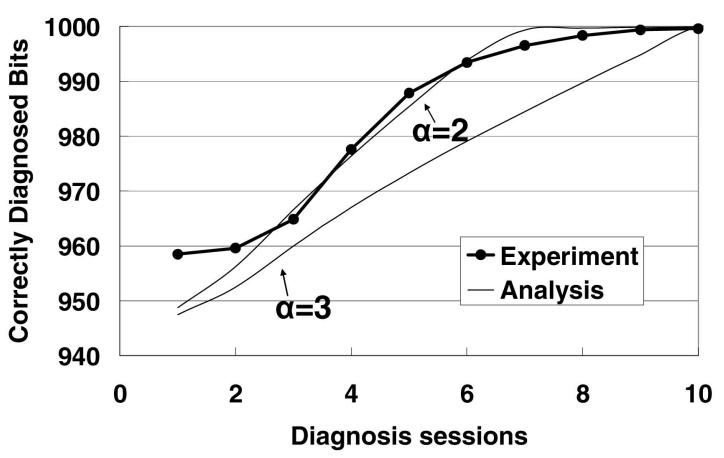

Fig. 14. Correctly diagnosed bits (clustered errors).

term is divided by two because the probability of selecting an unknown is a half. In addition to $v(s)$, the number of equations in the presence of unknowns, $q_{X}(s)$, is approximated by

$$
q_{X}(d, s) \approx q(d, s)-s r_{X}\left[1-2^{-s}\right],
$$

in which the last term represents the number of row equations discarded due to the selected unknowns. Besides $v_{X}(s)$ and $q_{X}(s)$, the other parts of $E C(s)$ remain unchanged.

Consider the third and fourth cases in which unknowns are clustered in a few scan chains. If no error is located in the same row as clustered unknowns, the diagnosis is not affected. If there are one or more errors in the same row as clustered unknowns, the row equation is useful only when none of the unknowns is selected. The probability of observing this row is $2^{-N x}$, where $N_{X}$ is the number of unknowns clustered in the same row.

\section{EXPERIMENTAL Results}

Experimental results are shown to demonstrate the effectiveness of the CPRS technique. The following experiments are performed on 1,000 scan cells in 10 scan chains ( $m=10$ and $l=100$ ) unless specified otherwise. Every one of the following experiments is performed on 10,000 randomly generated error matrices with various values of $r, c$, and e parameters. Although $10 \times 100$ does not represent a big CUT, it is already shown by previous analysis that the effectiveness of CPRS does not depend on the CUT size.

\subsection{Diagnosis without $X$}

\subsubsection{Clustered Errors}

Fig. 14 shows the expected number of correctly diagnosed bits versus the number of sessions. The thick line is the experimental data with ROI size $r=5$ and $c=10$. The error multiplicity is 40 -that is, 80 percent of the $\mathrm{ROI}$ is randomly filled with errors. The thin lines are calculated by (7) with parameters $\alpha=2$ and $\alpha=3$. The experimental results fit well with the analytical curve of $\alpha=2$ when $\mathrm{s}$ is small. CPRS achieves nearly perfect diagnosis after 10 sessions, which is predicted by the analytical curve of $\alpha=3$.

Table 2 shows the number of sessions to achieve a 99.9 percent correct diagnosis. The analytical results are calculated by (12) with $\alpha=3$. The analytical results match well with the experimental results. The results show that 
TABLE 2

Sessions Needed for 99.9 Percent Correct Diagnosis

\begin{tabular}{|c|c|c|c|c|}
\hline$r$ & $c$ & $e$ & Experiment & $\begin{array}{c}\text { Analytical } \\
\alpha=3\end{array}$ \\
\hline 2 & 10 & 15 & 7 & 5 \\
\hline 3 & 10 & 25 & 7 & 7 \\
\hline 4 & 10 & 35 & 8 & 9 \\
\hline 5 & 10 & 40 & 9 & 10 \\
\hline
\end{tabular}

CPRS provides good diagnosis results within 10 diagnosis sessions for an ROI of $5 \times 10$.

One notable feature of the $E C(s)$ curve in Fig. 14 is that the $E C(s)$ increases slowly when the number of sessions is small. In certain scenarios, the number of correctly diagnosed bits even decreases in the first few sessions. This phenomenon is because the number of error variables $v(s)$ increases faster than the number of equations $q(s)$ when $s$ is small. The session that suffers from the worst diagnosis results is obtained by taking the derivative of $E C_{\text {cluster }}(s)$ with respect to $s$. The $E C_{\text {cluster }}(s)$ derivative can be approximated by (17) with the $4^{-\mathrm{s}}$ terms ignored. Assuming that the $s$ term is greater than the $(1-s \ln 2)$ term, the session that produces the worst $E C_{\text {cluster }}(s)$ is further simplified to (18).

$$
\begin{aligned}
\frac{d E C_{\text {cluster }}(s)}{d s} \cong- & 2 c r 2^{-s} \ln 2+\frac{c+r}{\alpha}\left(1+2^{-s}-s 2^{-s} \ln 2\right) \\
& +2 e 2^{-s} \ln 2,
\end{aligned}
$$

$$
s_{\text {worst_EC }} \cong \log _{2}\left[\frac{\alpha(c r-e)}{c+r}\right]+0.47, \quad S_{\text {worst_EC }} \geq 0 .
$$

When errors are fully clustered $(e / c r=1)$, the $s_{\text {worst_EC }}$ is equal to zero. When the errors are half clustered, $(e / r c=0.5)$, the $s_{\text {worst_EC }}$ is around 3. Equation (18) indicates an encouraging fact that the $s_{\text {worst } E C}$ is not strongly dependent on the parameters $c, r$, and $e$ because of the $\log _{2}$ operator. For typical values of $r, c$, and $e$, the $s_{\text {worst_EC }}$ is no larger than four. Table 3 shows the diagnosis sessions that produce the worst EC(s). The experimental results are performed in a $5 \times 10$ ROI with various error density $(e / r c)$. The $s_{\text {worst_EC }}$ slowly increases as the error density decreases, but the worst EC session does not exceed four. The analytical results calculated from (18) match well with the experimental results.

TABLE 3

$\mathrm{S}_{\text {worst_EC }}(\mathrm{r}=5, \mathrm{c}=10)$

\begin{tabular}{|c|c|c|c|}
\hline \multirow{2}{*}{$e / c r$} & \multirow{2}{*}{ Experiment } & \multicolumn{2}{|c|}{ Analytical } \\
\cline { 3 - 4 } & & $\alpha=2$ & $\alpha=3$ \\
\hline 1 & 1 & 0 & 0 \\
\hline 0.8 & 1 & 1 & 1 \\
\hline 0.5 & 2 & 2 & 3 \\
\hline 0.25 & 2 & 3 & 3 \\
\hline 0.2 & 2 & 3 & 3 \\
\hline
\end{tabular}

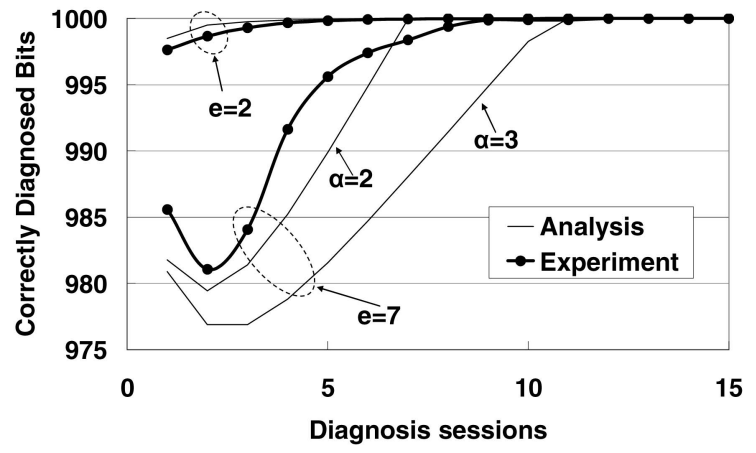

Fig. 15. Correctly diagnosed bits (scattered errors).

\subsubsection{Scattered Errors}

Fig. 15 shows the number of correctly diagnosed bits versus the number of sessions. The thick lines are the experimental results and the thin lines are calculated by (13). Seven spatial distributions in Table 1 are used. The error multiplicities are 2 and 7. As explained in the last section, a "hook" is observed when the number of sessions is small. The experimental data show that the worst diagnosis result occurs in the second session. Similarly to the clustered model, the worst-case diagnosis of the scattered model can be estimated by taking the derivative of $E C_{\text {scatter }}(s)$. The $s_{\text {worst_EC }}$ of the scattered model, however, cannot be expressed in simple analytical form due to the variety in spatial distributions.

Table 4 shows the average number of correctly diagnosed bits, wrong bits, and ambiguous bits. If a bit is uniquely solved, its diagnosis result is either correct or wrong. If a bit is not uniquely solved, its diagnosis result is ambiguous. The left three columns are obtained from the random diagnosis only and the right three columns are from the random diagnosis followed by one deterministic diagnosis session. After 15 random diagnosis sessions, every bit is correctly diagnosed, even in the presence of 15 errors (1.5 percent of total scan cells). The same experiments are performed using the previous technique [22]. The results show that only 993, 971, and 928 bits are correctly diagnosed for 2, 7, and 15 errors, respectively. In comparison, CPRS is more effective because of the row selection hardware.

The numbers of random sessions needed to achieve higher than 99.9 percent correct diagnosis for 2, 7, and 15 errors are 3, 8, and 15, respectively. The experimental results match the analytical equation (14), which predicts that 1 to 1.5 times $e$ sessions are required. The sessions that have the worst diagnosis for 2, 7, and 15 errors are 1, 2, and 4 , respectively. The results agree with the analysis, which states that the worst session is no more than four as long as the error multiplicity is reasonably small.

It is observed that the deterministic diagnosis helps to improve the diagnosis results, especially when $s$ is small. For the example of 15 errors, the number of correctly diagnosed bits is 914 if three random sessions are performed. The number can be improved to 930 if two random sessions plus one deterministic session are performed. The deterministic diagnosis, however, becomes less helpful when $s$ is large because the random diagnosis 
TABLE 4

Diagnosis Results of $10 \times 100$ (no X)

\begin{tabular}{|c|c|c|c|c|c|c|c|}
\hline \multirow{2}{*}{$\begin{array}{c}\text { \# of random } \\
\text { diagnosis } \\
\text { sessions }\end{array}$} & \multirow{2}{*}{ error multiplicity } & \multicolumn{3}{|c|}{ random only } & \multicolumn{3}{|c|}{ random +1 deterministic } \\
\hline & & correct & wrong & ambiguous & correct & wrong & ambiguous \\
\hline \multirow{3}{*}{1} & 2 & 997.7 & 1.2 & 1.1 & 998.1 & 1.2 & 0.7 \\
\hline & 7 & 986.1 & 5.4 & 8.4 & 987.5 & 6.2 & 6.3 \\
\hline & 15 & 960.8 & 11.9 & 27.4 & 955.4 & 11.9 & 32.7 \\
\hline \multirow{3}{*}{2} & 2 & 998.6 & 0.8 & 0.6 & 998.8 & 0.8 & 0.5 \\
\hline & 7 & 981.7 & 5.3 & 13.0 & 986.9 & 6.8 & 6.3 \\
\hline & 15 & 931.8 & 7.5 & 60.7 & 930.3 & 7.8 & 62.0 \\
\hline \multirow{3}{*}{3} & 2 & 999.3 & 0.4 & 0.3 & 999.3 & 0.4 & 0.2 \\
\hline & 7 & 984.5 & 6.6 & 8.9 & 990.4 & 6.9 & 2.7 \\
\hline & 15 & 914.6 & 5.2 & 80.2 & 915.5 & 6.1 & 78.4 \\
\hline \multirow{3}{*}{4} & 2 & 999.6 & 0.2 & 0.1 & 999.6 & 0.2 & 0.1 \\
\hline & 7 & 991.4 & 6.4 & 2.2 & 992.6 & 5.9 & 1.5 \\
\hline & 15 & 909.3 & 5.3 & 85.4 & 917.8 & 8.3 & 73.9 \\
\hline \multirow{3}{*}{5} & 2 & 999.8 & 0.1 & 0.1 & 999.8 & 0.1 & 0.1 \\
\hline & 7 & 995.1 & 4.0 & 0.9 & 995.5 & 3.8 & 0.8 \\
\hline & 15 & 929.2 & 11.5 & 59.2 & 956.7 & 14.2 & 29.1 \\
\hline \multirow{3}{*}{8} & 2 & 1000.0 & 0.0 & 0.0 & 1000.0 & 0.0 & 0.0 \\
\hline & 7 & 999.3 & 0.6 & 0.1 & 999.4 & 0.6 & 0.1 \\
\hline & 15 & 994.2 & 5.2 & 0.5 & 994.5 & 5.0 & 0.5 \\
\hline \multirow{3}{*}{10} & 2 & 1000.0 & 0.0 & 0.0 & 1000.0 & 0.0 & 0.0 \\
\hline & 7 & 999.9 & 0.1 & 0.0 & 999.9 & 0.1 & 0.0 \\
\hline & 15 & 998.8 & 1.1 & 0.1 & 998.8 & 1.1 & 0.1 \\
\hline \multirow{3}{*}{15} & 2 & 1000.0 & 0.0 & 0.0 & 1000.0 & 0.0 & 0.0 \\
\hline & 7 & 1000.0 & 0.0 & 0.0 & 1000.0 & 0.0 & 0.0 \\
\hline & 15 & 1000.0 & 0.0 & 0.0 & 1000.0 & 0.0 & 0.0 \\
\hline
\end{tabular}

already provides very good diagnosis results, leaving very small room for improvement.

Fig. 16 compares the diagnosis data volume of CPRS and the direct dumping technique. The $\mathrm{X}$-axis shows the total number of patterns applied $\left(P_{\text {total }}\right)$ and the $\mathrm{Y}$-axis shows the data volume. This plot is drawn according to (1) and (2) with $m=10$ and $l=100$. Two bottom lines indicate the CPRS data volume with two different $f$ parameters $\left(f=P_{\text {failing }} / P_{\text {total }}\right): 1$ percent and 10 percent. The number of random diagnosis sessions is 10 , which is chosen based

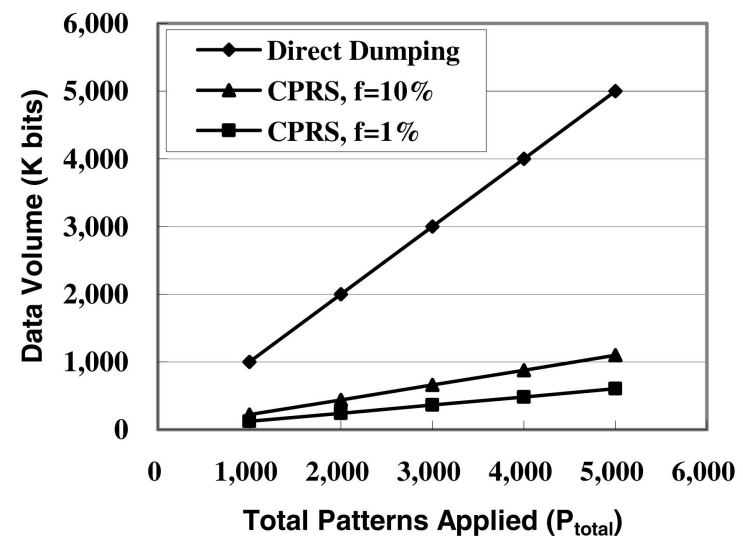

Fig. 16. Data volume versus $\mathrm{P}_{\text {total }}$. on the results of Table 4 . The top line represents the data volume of direct dumping. It can be seen that the CPRS data volume is only about one-fifth of the direct dumping, even when 10 percent of 5,000 patterns applied fail.

\subsubsection{Experiments on Benchmark Circuits}

To further validate the CPRS diagnosis technique, experiments are performed on three large ISCAS'89 and ITC'99 benchmark circuits. Fourteen randomly selected single stuck-at faults $\left(f_{1}\right.$ to $\left.f_{14}\right)$ are fault simulated in diagnosis mode and their complete faulty outputs are obtained (without stop on first failure). Regular SSF test patterns generated by a commercial ATPG are applied and the number of total patterns $\left(P_{\text {total }}\right)$ is shown in Table 5 . The number of failing patterns $\left(P_{\text {failing }}\right)$ and the number of errors are listed in the second and the third columns, respectively. All errors are correctly diagnosed in eight random sessions, except one error of $f_{6}$, which needs a deterministic diagnosis session. The last column is the percentage of CPRS data volume with respect to that of direct dumping. CPRS effectively reduced the data volume except for fault $f_{12}$, in which every failing pattern has a single error. As predicted by (1), the CPRS data volume is close to $1 / m$ when the number of errors is small (such as $f_{4}$, $\left.f_{10}, f_{11}\right)$. 
TABLE 5

Diagnosis Results of Benchmark Circuits

\begin{tabular}{|c|c|c|c|c|c|c|c|}
\hline \multirow{2}{*}{ Circuit } & \multirow{2}{*}{$\begin{array}{c}\text { injected } \\
\text { fault }\end{array}$} & \multirow{2}{*}{$P_{\text {failing }}$} & \multirow{2}{*}{$\begin{array}{l}\text { number } \\
\text { of errors }\end{array}$} & \multirow{2}{*}{$\begin{array}{l}\text { correctly } \\
\text { diagnosed }\end{array}$} & \multicolumn{3}{|c|}{ Data Volume } \\
\hline & & & & & direct dump & CPRS & CPRS/DD \\
\hline \multirow{5}{*}{$\begin{array}{c}\mathrm{b} 19 \\
230 \mathrm{~K} \text { gates } \\
m=21 \\
l=328 \\
P_{\text {total }}=931\end{array}$} & $f_{l}$ & 395 & 395 & $100 \%$ & $6,560,000$ & 1447,680 & $22.1 \%$ \\
\hline & $f_{2}$ & 16 & 23 & $100 \%$ & $6,560,000$ & 392,544 & $6.0 \%$ \\
\hline & $f_{3}$ & 77 & 112 & $100 \%$ & $6,560,000$ & 562,368 & $8.6 \%$ \\
\hline & $f_{4}$ & 2 & 2 & $100 \%$ & $6,560,000$ & 353,568 & $5.4 \%$ \\
\hline & $f_{5}$ & 31 & 31 & $100 \%$ & $6,560,000$ & 434,304 & $6.6 \%$ \\
\hline \multirow{5}{*}{$\begin{array}{c}\mathrm{s} 9234 \\
5.6 \mathrm{~K} \text { gates } \\
m=5 \\
l=42 \\
P_{\text {total }}=614\end{array}$} & $f_{6}$ & 104 & 245 & $99.6 \%$ & 215,000 & 87,936 & $40.9 \%$ \\
\hline & $f_{7}$ & 96 & 104 & $100 \%$ & 215,000 & 84,864 & $39.5 \%$ \\
\hline & $f_{8}$ & 24 & 32 & $100 \%$ & 215,000 & 57,216 & $26.6 \%$ \\
\hline & $f_{9}$ & 39 & 47 & $100 \%$ & 215,000 & 62,976 & $39.3 \%$ \\
\hline & $f_{10}$ & 4 & 12 & $100 \%$ & 215,000 & 49,536 & $23.0 \%$ \\
\hline \multirow{4}{*}{$\begin{array}{c}\mathrm{s} 38584 \\
19 \mathrm{~K} \text { gates } \\
m=5, l=285 \\
P_{\text {total }}=1,070\end{array}$} & $f_{11}$ & 2 & 3 & $100 \%$ & $1,430,000$ & 295,656 & $20.7 \%$ \\
\hline & $f_{12}$ & 549 & 549 & $100 \%$ & $1,430,000$ & 1569,072 & $109.7 \%$ \\
\hline & $f_{13}$ & 78 & 78 & $100 \%$ & $1,430,000$ & 472,584 & $33.0 \%$ \\
\hline & $f_{14}$ & 207 & 414 & $100 \%$ & $1,430,000$ & 772,896 & $54.0 \%$ \\
\hline
\end{tabular}

TABLE 6

Diagnosis Results of $10 \times 100$ (Seven Errors, with $\mathrm{X}$ )

\begin{tabular}{|c|c|c|c|c|c|c|c|}
\hline & & \multicolumn{3}{|c|}{ Scattered X } & \multicolumn{3}{|c|}{ Clustered X } \\
\hline $\begin{array}{l}\text { random } \\
\text { sessions }\end{array}$ & $\begin{array}{l}\text { unknown } \\
\text { multiplicity }\end{array}$ & correct & wrong & Amb. & correct & wrong & Amb. \\
\hline \multirow{3}{*}{1} & 1 & 985.6 & 5.4 & 9.0 & 985.7 & 5.5 & 8.9 \\
\hline & 5 & 986.7 & 6.0 & 7.3 & 986.6 & 5.9 & 7.5 \\
\hline & 10 & 986.4 & 6.3 & 7.4 & 986.6 & 6.0 & 7.4 \\
\hline \multirow{3}{*}{2} & 1 & 982.1 & 5.5 & 12.4 & 981.6 & 5.3 & 13.0 \\
\hline & 5 & 984.2 & 6.5 & 9.3 & 984.0 & 6.3 & 9.7 \\
\hline & 10 & 985.8 & 7.1 & 7.1 & 984.5 & 6.6 & 8.9 \\
\hline \multirow{3}{*}{3} & 1 & 984.8 & 7.0 & 8.1 & 984.1 & 6.8 & 9.2 \\
\hline & 5 & 985.4 & 7.9 & 6.7 & 985.7 & 8.0 & 6.3 \\
\hline & 10 & 986.7 & 8.5 & 4.8 & 986.4 & 8.6 & 5.0 \\
\hline \multirow{3}{*}{4} & 1 & 990.8 & 7.0 & 2.2 & 991.0 & 6.8 & 2.1 \\
\hline & 5 & 990.2 & 7.9 & 1.9 & 990.2 & 8.1 & 1.8 \\
\hline & 10 & 989.4 & 8.7 & 1.9 & 989.7 & 8.7 & 1.7 \\
\hline \multirow{3}{*}{5} & 1 & 994.4 & 4.7 & 0.9 & 994.3 & 4.8 & 0.9 \\
\hline & 5 & 993.2 & 6.0 & 0.8 & 992.7 & 6.5 & 0.9 \\
\hline & 10 & 991.6 & 7.5 & 0.9 & 991.7 & 7.5 & 0.8 \\
\hline \multirow{3}{*}{8} & 1 & 999.2 & 0.8 & 0.1 & 998.5 & 1.3 & 0.2 \\
\hline & 5 & 997.6 & 2.2 & 0.2 & 996.8 & 3.0 & 0.2 \\
\hline & 10 & 995.4 & 4.3 & 0.3 & 995.5 & 4.4 & 0.1 \\
\hline \multirow{3}{*}{10} & 1 & 999.7 & 0.3 & 0.0 & 999.5 & 0.5 & 0.1 \\
\hline & 5 & 998.2 & 1.7 & 0.1 & 997.8 & 2.1 & 0.1 \\
\hline & 10 & 996.2 & 3.5 & 0.2 & 996.2 & 3.8 & 0.1 \\
\hline \multirow{3}{*}{15} & 1 & 999.8 & 0.2 & 0.0 & 999.8 & 0.2 & 0.0 \\
\hline & 5 & 998.8 & 1.1 & 0.1 & 998.2 & 1.7 & 0.1 \\
\hline & 10 & 997.2 & 2.6 & 0.2 & 996.6 & 3.3 & 0.1 \\
\hline
\end{tabular}

\subsection{Diagnosis with Unknowns}

Table 6 shows the diagnosis results with seven errors and various unknown multiplicities. Unknown multiplicity means the number of unknowns injected. Two types of experiments are performed: scattered $X$ and clustered $X$. In the former experiment, the locations of $\mathrm{Xs}$ are randomly generated, assuming a scattered spatial distribution. In the latter experiment, the unknowns are assumed to be clustered into two scan chains; the other eight scan chains are free of unknowns. This is to model the real circuit scenario in which only a few scan chains generate unknowns [1]. It can be seen from the results that CPRS is equally effective for both experiments. More than 99 percent of the 10,000 scan cells are correctly diagnosed, even in the

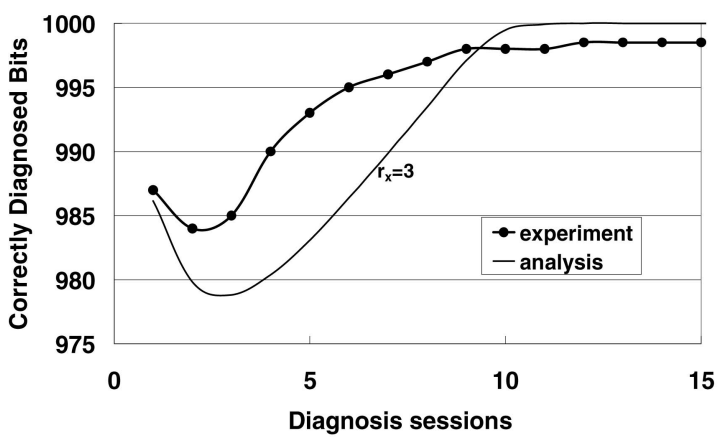

Fig. 17. Correctly diagnosed bits (seven errors, five X). 
TABLE 7

Solving RS-LFSR Seeds (1,000,000 Cases per Row)

\begin{tabular}{|c|c|c|c|}
\hline $\begin{array}{c}\text { RS-LFSR } \\
\text { length }\end{array}$ & $\begin{array}{c}\text { Care } \\
\text { bits }\end{array}$ & $\begin{array}{c}\text { CPU } \\
\text { time(sec) }\end{array}$ & $\begin{array}{c}\text { Solved } \\
\text { cases }\end{array}$ \\
\hline 16 & 6 & 21.7 & 999,832 \\
\hline 16 & 12 & 28.8 & 971,489 \\
\hline 32 & 6 & 31.2 & 999,995 \\
\hline 32 & 12 & 40.5 & 999,203 \\
\hline
\end{tabular}

presence of 1 percent unknowns. Unfortunately, previous BIST diagnosis techniques, such as [3], [8], [15], [16], do not consider so many unknowns, so there is no direct comparison available so far.

Fig. 17 plots the number of correctly diagnosed bits in the presence of seven errors and five unknowns. The thick dots are from experiments and the thin line is calculated with the modifications in (15) and (16) taken into account. The parameter $r_{X}$ used in the figure is three because approximately half of the seven errors can share a row with unknowns. The analytical result is pessimistic when $s$ is small, but optimistic when $s$ is large. Compared with the $E C(s)$ curve without $\mathrm{X}$, the $E C(s)$ curve with $\mathrm{X}$ deviates farther away from the experimental result. This is understandable since the location of unknowns is simply modeled by a single parameter $r_{x}$.

\section{Discussions}

\subsection{RS-LFSR Seed Solver}

The deterministic RS-LFSR seed can be solved from the $S^{M D}$ by a mod-2 linear equation solver. To demonstrate the efficiency of the seed solving, a Gauss-Jordan elimination algorithm is implemented in about 500 lines of $\mathrm{C}++$ program. Table 7 shows the data from four experiments performed on a 32-bit and a 16-bit RS-LFSR with 1,000 error variables in $S^{M D}$. In every experiment, $1,000,000 S^{M D}$ with a specified number of care bits (6 or 12) are randomly generated. The number of care bits represents the number of unsolved variables after random diagnosis. The number of care bits is based on the results of Table 4, in which the number of unsolved bits is less than six after eight random sessions. The unspecified bits are filled with don't cares. On a $2.66 \mathrm{GHz}$ Intel PC, the total CPU time for 1,000,000 cases is less than a minute, which shows that the deterministic RS-LFSR seeds can be easily solved in a short time. The last column shows the number of solved cases (out of $1,000,000$ ).
TABLE 9 RS-LFSR Area

\begin{tabular}{|c|c|c|c|}
\hline Stages & $\begin{array}{c}\text { Scannable } \\
\text { FF }\end{array}$ & XOR & $\begin{array}{c}\text { Total Area } \\
\text { (NAND) }\end{array}$ \\
\hline 8 & 8 & 2 & 57 \\
\hline 16 & 16 & 2 & 110 \\
\hline 32 & 32 & 2 & 213 \\
\hline
\end{tabular}

A case is solved if an RS-LFSR seed is found to satisfy the care bits of the $S^{M D}$. The success rate is very high as long as the RS-LFSR stage number is much larger than the number of care bits.

\subsection{Hardware Overhead}

Table 8 compares the diagnosis circuitry area overhead of several techniques. The number of flip-flops, XOR, and AND gates is derived from the original papers. The total area is expressed in the number of equivalent NAND gates as a function of $m$ (number of scan chains). The conversion of a cell area is based on the numbers in the TSMC $0.18 \mu \mathrm{m}$ standard cell library. The CPRS costs about 20 NAND gates per scan chain, which is approximately on the same order as the other techniques. For convenience of comparison, some typical numbers are assumed for the previous techniques.

The area overhead of 8,16 , and 32-stage RS-LFSR is shown in Table 9. Because the seeds have to be loaded into the RS-LFSR, the flip-flops are scannable. Also, RS-LFSR needs two 2-input XOR for the feedback. The total equivalent gate count is calculated in the last column. According to the probability analysis in [28], the 32-stage RS-LFSR is able to handle 12 unsolved error variables in the deterministic diagnosis, which is good enough for most diagnosis scenarios. From this table, it can be seen that RS-LFSR is an inexpensive solution for diagnosis in terms of area, especially for large design under test.

\section{SUMmarY}

The CPRS is an effective X-tolerant BIST diagnosis technique. The row selection LFSR randomly selects scan outputs from multiple scan chains. The column parity and the row parity of the selected scan outputs are observed after every scan cycle and every scan unload, respectively. Experimental data show that CPRS is very effective, even in the presence of 1 percent unknowns. Two models are proposed to evaluate the performance of CPRS analytically. The probability analysis shows that the CPRS effectiveness

TABLE 8

Comparison of Area Overhead

\begin{tabular}{|c|r|r|r|r|}
\hline Techniques & \multicolumn{1}{|c|}{ FF } & \multicolumn{1}{|c|}{ XOR } & \multicolumn{1}{|c|}{ NAND } & Total Area (NAND) \\
\hline Random [16] & $\log _{2} b+m+k$ & $K \log _{2} b+m+k$ & $m\left(\log _{2} b+1\right)$ & $32 m+23^{*}$ \\
\hline CC3 [23] & 32 & $3 m$ & 1 & $182+8 m$ \\
\hline CC7 [23] & 32 & $7 m$ & 1 & $182+19 m$ \\
\hline Parity [22] & $m$ & $2 m$ & 0 & $11 m$ \\
\hline CPRS & $2 m$ & $3 m$ & $m$ & $20 m$ \\
\hline
\end{tabular}

*assume $k=m, \mathrm{~b}=16$ 
does not depend on the CUT size. The analysis provides good insight into the diagnosis effectiveness and the analytical results match well with the experimental data.

\section{ACKNOWLEDGMENTS}

This research is partly sponsored by the National Science Council of Taiwan under contract 95-2220-E-002 and by the Ministry of Economy of Taiwan under contract 94-EC-17-AS1-00 (HOY project). The idea of CPRS was first proposed at the International Test Conference in 2005.

\section{REFERENCES}

[1] J. Rajski, J. Tyszer, S.M. Reddy, and C. Wang, "Finite Memory Test Response Compactors for Embedded Test Applications," IEEE Trans. Computer-Aided Design of Integrated Circuits and Systems, vol. 24, no. 4, pp. 622-634, Apr. 2005.

[2] P. Wohl, J.A. Waicukauski, S. Patel, and M.B. Amin, "Efficient Compression and Application of Deterministic Patterns in a Logic BIST Architecture," Proc. Design Automation Conf., pp. 566-569, 2003.

[3] J. Ghosh-Dastidar and N.A. Touba, "Rapid and Scalable Diagnosis Scheme for BIST Environments with a Large Number of Scan Chains," Proc. IEEE VLSI Test Symp., pp. 79-85, 2000.

[4] J.A. Waicukauski and E. Lindbloom, "Failure Diagnosis of Structured VLSI," IEEE Design and Test of Computers, vol. 6, no. 4, pp. 49-60, Aug. 1989.

[5] W.H. McAnney and J. Savir, "There Is Information in Faulty Signature," Proc. IEEE Int'l Test Conf., pp. 630-636, 1987.

[6] J.C. Chan and J.A. Abraham, "A Study of Faulty Signatures Using a Matrix Formulation," Proc. IEEE Int'l Test Conf., pp. 553-561, 1990.

[7] R.C. Aitken and V.K. Agarwal, "A Diagnosis Method Using Pseudo-Random Vectors without Intermediate Signatures," Proc. IEEE Int'l Conf. Computer-Aided Design, pp. 574-577, 1989.

[8] J. Savir, "Salvaging Test Windows in BIST Diagnostics," Proc. IEEE VLSI Test Symp., pp. 416-425, 1997.

[9] J. Rajski and J. Tyszer, "On the Diagnostic Properties of Linear Feedback Shift Registers," IEEE Trans. Computer-Aided Design of Integrated Circuits and Systems, vol. 10, no. 10, pp. 1316-1322, Oct. 1991.

[10] M.G. Karpovsky and S.M. Chaudhry, "Design of Self-Diagnostic Boards by Multiple Signature Analysis," IEEE Trans. Computers, vol. 42, no. 9, pp. 1035-1044, Sept. 1993.

[11] T.R. Damarla, C.E. Stroud, and A. Sathaye, "Multiple Error Detection and Identification via Signature Analysis," J. Electronic Testing: Theory and Applications (JETTA), vol. 7, no. 3, pp. 193-207, 1995.

[12] J. Savir and W.H. McAnney, "Identification of Failing Tests with Cycling Registers," Proc. IEEE Int'l Test Conf., pp. 322-328, 1988.

[13] J. Ghosh-Dastidar, D. Das, and N.A. Touba, "Fault Diagnosis in Scan-Based BIST Using Both Time and Space Information," Proc. IEEE Int'l Test Conf., pp. 95-102, 1999.

[14] T.R. Damarla, W. Su, M.J. Chung, C.E. Stroud, and G.T. Michael, "Built-In Self Test Scheme for VLSI," Proc. Asia and South Pacific Design Automation Conf., pp. 217-222, 1995.

[15] Y. Wu and S. Adham, "BIST Fault Diagnosis in Scan-Based VLSI Environments," Proc. IEEE Int'l Test Conf., pp. 48-57, 1996.

[16] J. Rajski and J. Tyszer, "Fault Diagnosis in Scan-Based BIST," Proc. IEEE Int'l Test Conf., pp. 894-902, 1997.

[17] I. Bayraktaroglu and A. Orailoglu, "Deterministic Partitioning Techniques for Fault Diagnosis in Scan-Based BIST," Proc. IEEE Int'l Test Conf., pp. 273-282, 2000.

[18] C. Liu and K. Chakrabarty, "Failing Vector Identification Based on Overlapping Intervals of Test Vectors in a Scan-BIST Environment," IEEE Trans. Computer-Aided Design of Integrated Circuits and Systems, vol. 22, no. 5, pp. 593-604, May 2003.

[19] P. Wohl, J.A. Waicukauski, and S. Patel, "Scalable Selector Architecture for X-Tolerant Deterministic BIST," Proc. Design Automation Conf., pp. 934-939, 2004.

[20] R.C. Tekumalla, "On Reducing Aliasing Effects and Improving Diagnosis of Logic BIST Failures," Proc. IEEE Int'l Test Conf., pp. 737-744, 2003.
[21] S. Mitra and K.S. Kim, "X-Compact: An Efficient Response Compaction Technique for Test Cost Reduction," Proc. IEEE Int'l Test Conf., pp. 311-320, 2002.

[22] O. Sinanoglu and A. Orailoglu, "Compacting Test Responses for Deeply Embedded SoC Cores," IEEE Design and Test of Computers, vol. 20, no. 4, pp. 22-30, July/Aug. 2003.

[23] J. Rajski, J. Tyszer, and S.M. Reddy, "Convolutional Compaction of Test Responses," Proc. IEEE Int'l Test Conf., pp. 745-754, 2003.

[24] G. Mrugalski, A. Pogiel, J. Rajski, J. Tyszer, and C. Wang, "Fault Diagnosis in Designs with Convolutional Compactors," Proc. IEEE Int'l Test Conf., pp. 498-507, 2004.

[25] W.-T. Cheng, K.-H. Tsai, Y. Huang, N. Tamarapalli, and J. Rajski, "Compactor Independent Direct Diagnosis," Proc. Asian Test Symp., pp. 204-209, 2004

[26] H.M. Lin and J.C.M. Li, "Column Parity and Row Selection (CPRS): A BIST Diagnosis Technique for Multiple Errors in Multiple Scan Chains," Proc. IEEE Int'l Test Conf., p. 42.3, 2005.

[27] A.A. Al-Yamani and E.J. McCluskey, "Seed Encoding with LFSRs and Cellular Automata," Proc. Design Automation Conf., pp. 560565, 2003.

[28] B. Koenemann, "LFSR-Coded Test Patterns for Scan Designs," Proc. European Test Conf., pp. 237-242, 1991.

[29] I. Koren, Z. Koren, and C.H. Stapper, "A Statistical Study of Defect Maps of Large Area VLSI IC's," IEEE Trans. VLSI Systems, vol. 2, no. 2, pp. 249-256, June 1994.

[30] J.C.-M. Li and E.J. McCluskey, "Diagnosis of Resistive and Stuckopen Defects in Digital CMOS IC," IEEE Trans. Computer-Aided Design, vol. 24, no. 11, pp. 1748-1759, 2005.

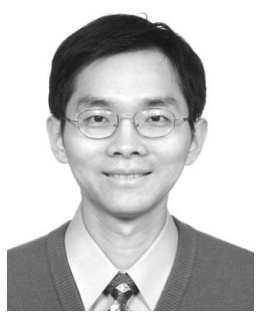

James Chien-Mo Li (M'02) received the BSEE degree in 1993 from National Taiwan University, Taipei, Taiwan. He received the MSEE and PhD degrees in electrical engineering from Stanford University in 1997 and 2002, respectively. He is currently an associate professor in the Graduate Institute of Electronics Engineering, National Taiwan University, Taipei, Taiwan. His research interests include design for testability, built-in self-test, defect-based testing, and fault diagnosis. He is a member of the IEEE.

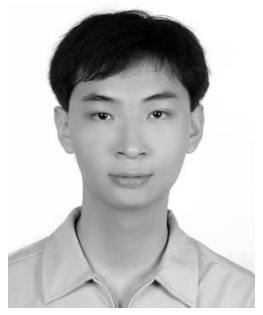

Hung-Mao Lin received the BSEE degree in 2003 and the MSEE degree in 2005 from National Taiwan University, Taipei, Taiwan. His current research interests include circuit diagnosis, circuit design, and circuit architecture.

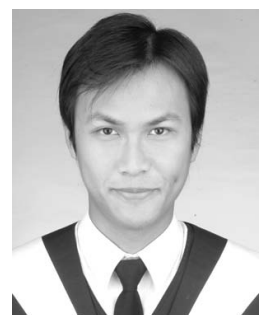

Fang-Min Wang received the bachelor's degree in mathematics from Fu-Jen Catholic University, Taipei, Taiwan, in 2005. He is currently a master's student in the Graduate Institute of Electronics Engineering, National Taiwan University. His research interests include logic BIST, fault diagnosis, and test response compression.

$\triangleright$ For more information on this or any other computing topic, please visit our Digital Library at www.computer.org/publications/dlib. 